\title{
EFEITO DE PRÉ-TRATAMENTOS NA TAXA DE SECAGEM E NA QUALIDADE DA MADEIRA: UMA REVISÃO
}

\section{Nadia Helena Bianchini'; Roberta Rodrigues Roubuste ${ }^{2}$; Guilherme Valcorte ${ }^{3}$; Elio José Santini ${ }^{4}$}

\section{RESUMO}

A secagem é uma etapa do processamento da madeira sólida essencial por agregar valor ao produto final, possuindo relevância econômica para as empresas, visto que trás benefícios econômicos. Desta forma, na indústria de transformação da madeira é necessário conhecer as técnicas que possibilitem a diminuição do tempo de secagem, visando reduzir o consumo de energia do processo ao mesmo tempo que se diminui dos custos, para alavancar os lucros das organizações. Desta forma, o objetivo deste estudo foi elaborar uma revisão de literatura acerca dos efeitos do uso de pré-tratamentos na taxa de secagem e na qualidade de madeiras submetidas a estes processos. Buscou-se estudos sobre o efeito tratamentos com vapor, tratamento com água quente e congelamento na taxa de secagem e na qualidade da madeira seca. Os pré-tratamentos pesquisados influenciam na secagem da madeira, cada um com suas peculiariedades, agregando valor a madeira seca.

Palavras-chave: Pré-tratamento da madeira; Secagem da madeira; Defeitos da madeira; Tecnologia de produtos florestais.

Eixo Temático: Sociedade e Ambiente (SA).

\section{INTRODUÇÃO}

Essencial para a manutenção da qualidade da madeira, a secagem é uma das etapas do processamento da madeira sólida responsável por agregar valor ao produto final. Peng et al. (2012) afirmam que a secagem é um passo fundamental na fabricação de produtos madeireiros, que além de utilizar muita energia, é um

\footnotetext{
1 Doutoranda do Programa de Pós-graduação em Engenharia Florestal da Universidade Federal de Santa Maria (UFSM); nadia bianchini@hotmail.com.

2 Mestranda do Programa de Pós-graduação em Engenharia Florestal da UFSM; robertaroubuste@hotmail.com.

${ }^{3}$ Doutorando do Programa de Pós-graduação em Engenharia Florestal da UFSM; gvalcorte@gmail.com.

${ }^{4}$ Professor Titular do Departamento de Ciências Florestais da UFSM; ejsantini@gmail.com.
} 
processo demorado. Estes autores relatam ainda que tanto a taxa de secagem quanto o ciclo de secagem afetam os benefícios econômicos das empresas. Adicionalmente, reduz a massa e os custos de transporte e manuseio, aumenta a resistência mecânica, além de reduzir a instabilidade dimensional da madeira (MARTINS, 1988).

Dentre as espécies de madeira existentes, aquelas de baixa permeabilidade apresentam diversas dificuldades durante a manufatura, oriundas de um longo e ineficiente tempo de secagem, o que torna o processo oneroso e com alto consumo de energia (HE et al., 2017). Estes mesmos autores afirmam também que um longo tempo de secagem pode ser associado também a geração de defeitos dentro da madeira, devido ao alto estresse interno, que introduz custos adicionais para a recuperação da madeira serrada.

Neste cenário, Talgatti et al. (2016) afirmam que na indústria de transformação da madeira, é necessário conhecer as técnicas que possibilitem a diminuição do tempo de secagem, visando reduzir o consumo de energia do processo concomitante a diminuição dos custos, para aumentar os lucros das organizações. Ao mesmo tempo em que se otimize o processo, é imprescindível que se preserve ou aprimore as características da madeira, evitando a ocorrência de defeitos ocasionados pela secagem.

O tratamento térmico da madeira, por exemplo, é um método de aquecimento a temperaturas entre $160-260^{\circ} \mathrm{C}$, que induz mudanças na composição química da madeira por hidrólise térmica para aumentar as propriedades físico-mecânicas e a resistência à deterioração da madeira (MILITZ, 2002). Esta é considerada a principal faixa de temperatura do tratamento térmico, onde a madeira sofre várias alterações nos componentes químicos, por meio da degradação dos extrativos ou componentes da parede celular (PARK et al., 2017). As mudanças químicas que ocorrem nesta faixa de temperatura são principalmente devido a reações auto catalíticas dos constituintes da parede celular (TJEERDSMA; MILITZ, 2005) e dependem do tempo, da temperatura, da espécie e o tipo de tratamento térmico, ou seja, o meio de transferência de calor (KIM et al., 2012; WANG et al., 2016).

Park et al. (2017) afirmam que abaixo de $150^{\circ} \mathrm{C}$, a madeira inicia a secagem 
por meio da perda de água livre e finaliza com a "água ligada". Inicialmente, a hemicelulose, que é o componente da parede celular mais sensível ao aquecimento, é degradada por desacetilação, seguido pela despolimerização catalisada pelo ácido acético liberado. Já a celulose é menos afetada pelo tratamento térmico, provavelmente devido sua natureza cristalina. O tratamento térmico causa também a clivagem da ligação $\beta-O-4$ da lignina, enquanto que extrativos como ácidos resínicos desapareceram da madeira a temperaturas acima de $200{ }^{\circ} \mathrm{C}$. Acima de $260{ }^{\circ} \mathrm{C}$, a madeira inicia a carbonização com a geração de produtos de pirólise, como dióxido de carbono (ESTEVES; PEREIRA, 2009; WIKBERG; MAUNU, 2004; NUOPPONEN et al., 2003).

Dentre as vantagens de se tratar a madeira termicamente estão a melhora da estabilidade dimensional, com o aumento da hidrofobicidade, a equalização da coloração da superfície e aumento da resistência ao apodrecimento (PARK et al., 2017). Devido a essas vantagens, diversos métodos de tratamento térmico foram desenvolvidos principalmente na Europa, e as propriedades da madeira tratada termicamente por cada método continuam sendo investigadas (REPELLIN; GUYONNET, 2005; TUONG; LI, 2011).

Neste contexto, os pré-tratamentos térmicos surgem como uma alternativa para reduzir o tempo de secagem e a ocorrência de defeitos na madeira. Por este motivo, este tem sido o foco de pesquisas sobre secagem de madeiras do gênero Eucalyptus, por exemplo, que visam reduzir o tempo e os custos de secagem, aumentando a qualidade da madeira seca (SEVERO; TOMASELLI, 2001). O efeito positivo ou negativo do uso de pré-tratamentos antecedente à secagem da madeira vai depender da intensidade e da espécie que está sendo tratada (GLOSSOP, 1994). O congelamento, imersão em água quente e vaporização são alguns exemplos de pré-tratamentos e são considerados formas de facilitar a secagem da madeira (GLOSSOP, 1994; REZENDE et al., 2015).

\section{METODOLOGIA}

Para o desenvolvimento deste estudo, realizou-se uma revisão de literatura em periódicos nacionais e internacionais, nas plataformas Periódicos Capes, Scopus 
e Google acadêmico, além de livros disponíveis sobre o tema de estudo. Para isso, utilizou-se as palavras-chave "tratamentos com vapor"; "tratamento com água quente"; "congelamento"; "taxa de secagem" e "qualidade da madeira".

\section{RESULTADOS E DISCUSSÕES}

\subsection{TRATAMENTOS COM VAPOR}

A vaporização na madeira é uma técnica que consiste na aplicação de vapor aquecido a alta temperatura e umidade relativa por determinado tempo. O vapor superaquecido, que tem uma temperatura acima do ponto de ebulição da água, vem sendo utilizado para a secagem desde 1900 e é conhecido por ter sido utilizado na indústria em 1930, na Alemanha (PARK et al.,2012). A vaporização preliminar à secagem (pré-vaporização) é atrativa por se tratar de uma técnica que pode ser adotada como método comercial, devido a pouca ou nenhuma mudança necessária em termos de equipamentos ou operação básica em uma indústria (SEVERO; TOMASELLI, 2001).

A aplicação desta técnica é feita com a finalidade de reduzir os níveis de tensões de crescimento, esterilizar a madeira, aumentar a sua permeabilidade, reduzir a instabilidade dimensional, reduzir o teor de umidade inicial antes da secagem propriamente dita, reduzir os gradientes de umidade, reduzir o tempo de secagem e aumentar a taxa de secagem. Entretanto, sua aplicação pode implicar em alto custo energético e pode causar alterações na coloração da madeira, embora a mudança de cor possa ser um aspecto positivo sob um ponto de vista estético, agregando valor a madeira, dependendo do seu uso (REZENDE et al., 2015; CALONEGO; SEVERO, 2005).

O tratamento de secagem e tratamento térmico utilizando vapor superaquecido a alta temperatura e alta pressão é chamado de "tratamento combinado" e é utilizado para secar madeira verde (PARK et al., 2017). Diversos autores já avaliaram diferentes variáveis relacionadas ao tratamento combinado. Park et al. (2012) mensuraram as variações das propriedades físicas e mecânicas da madeira de pinheiro coreano tratadas termicamente com vapor superaquecido a $170{ }^{\circ} \mathrm{C}$ e $0,4 \mathrm{Mpa}$, por 10 horas. Já Miao e Zhang (2009) avaliaram o efeito do pré- 
tratamento com vapor sobre a permeabilidade da madeira e revelaram uma melhoria na permeabilidade radial e tangencial, que implica em um aumento na taxa de secagem e uma diminuição no tempo necessário (PENG et al., 2012).

Em tratamento combinado com pinheiro verde utilizando vapor superaquecido a várias temperaturas e pressão em escala laboratorial, Park et al. (2014) determinaram as condições adequadas de temperatura e pressão de vapor superaquecido. Estes autores relataram que, como o tratamento combinado realiza simultaneamente os processos de secagem e tratamento térmico, pode diminuir o tempo e a energia necessária para o processo de secagem, além de prevenir incêndios. O uso desta técnica facilita o fluxo em função do aumento da temperatura da madeira e aumento do coeficiente de difusão. A vaporização promove uma hidrolização parcial da membrana das pontoações, aumentando a permeabilidade e modificando a estrutura da membrana das pontoações, o que dilata e facilita a remoção de água durante a secagem (CHOONG et al., 1999; DIAZ-VAZ et al., 1998).

Os polímeros amorfos da madeira atingem temperaturas de transição vítrea, tornando-os mais fluidos e flexíveis. Com o amolecimento da estrutura física da madeira, decorrente de maior fluidos molecular, há um alívio de tensões internas, o reduzindo os defeitos de secagem. Apesar dos benefícios e da proposição atual da técnica para diferentes espécies, ainda não há um consenso sobre a duração, o momento de aplicação e as condições do tratamento para cada espécie (REZENDE et al., 2015; AGUIAR; PERRÉ, 2005; CALONEGO; SEVERO, 2005).

O método de secagem com vapor superaquecido possibilita também uma economia de energia na secagem, já que é fácil condensar o vapor superaquecido utilizado. Apresenta também a vantagem de ter um baixo escape de substâncias perigosas, um alto nível de segurança devido à ausência de oxidação e combustão, além dos efeitos adicionais de esterilização ou desodorização, quando se utiliza vapor de alta temperatura. E, por fim, o método apresenta uma taxa de secagem rápida, além de ser fácil de combinar com outros métodos (PARK et al,2012). Em um estudo realizado por KANG et al. (2014), amostras de madeira de Pseudotsuga 
menziesii tratadas com vapor apresentaram uma taxa de secagem mais rápida e a tensão de secagem foi mais branda em relação ao tratamento controle.

\subsection{TRATAMENTO COM ÁGUA QUENTE}

O pré-aquecimento em água consiste na imersão da madeira em um meio com água quente, utilizando temperaturas e períodos variáveis. O pré-tratamento térmico imersão em água quente possibilita a remoção ou redistribuição dos extrativos responsáveis por causar alterações na estrutura interna da madeira, promovendo um aumento no coeficiente de difusão e na taxa de secagem (CHOONG et al., 1999). Da mesma forma como o pré-tratamento com vaporização, o banho em água quente também modifica o comportamento da secagem.

O conceito deste tipo de pré-tratamento é bastante variável. Segundo Vivian et al. (2011), o tratamento térmico de aquecimento consiste na imersão da madeira em água quente, utilizando-se temperaturas e períodos variáveis. De acordo com Chafe (1992), o aquecimento em água produz mudanças nas propriedades físicas e químicas da madeira e a extensão dessas mudanças depende da temperatura e da duração do pré-tratamento. De acordo com Haslett e Kininmonth (1986), o banho em água quente causa relocação parcial de polifenóis, resultando em substancial redução no tempo de secagem. A vantagem do banho em água quente é o aumento da taxa de secagem inicial sem acrescentar defeitos na madeira.

\subsection{CONGELAMENTO}

Os primeiros estudos utilizando o pré-congelamento como pré-tratamento de secagem da madeira são datados da década de sessenta e foram desenvolvidos nos Estados Unidos. Estas pesquisas já demostravam os benefícios na secagem da madeira com o uso desse pré-tratamento, com o aumento da taxa de secagem e redução de defeitos de secagem na madeira (OLIVEIRA et al., 2003).

O pré-tratamento de congelamento, de acordo com llic (1995), tem sido utilizado como pré-tratamento de secagem para madeiras de coníferas e folhosas de regiões temperadas e tropicais. De acordo com Vivian et al. (2011), temperaturas próximas a $-20^{\circ} \mathrm{C}$ são mais apropriadas para o congelamento da madeira, embora 
algumas espécies respondam melhor a temperaturas mais negativas, com durações que variam entre 12 a $24 \mathrm{~h}$. Cooper (1972) relata que o pré-congelamento modifica a relação água-madeira, o que pode acelerar o processo de secagem sem a ocorrência de defeitos no material. Illic (1999) corrobora ao afirmar que o précongelamento da madeira pode reduzir os defeitos durante a secagem, aumentar a taxa de secagem e também reduzir sua retratibilidade.

Cooper et al. (1970) relatam que é significante e favorável a alteração da relação água-madeira causada pelo pré-congelamento. Como o efeito ocorre acima do ponto de saturação das fibras, o pré-congelamento aumenta a massa de fluxo líquido, que pode ser expressa como permeabilidade da madeira. Adicionalmente, o pré-congelamento da madeira é utilizado na área de conservação e restauração de obras de arte, e seu uso em algumas etapas do processo industrial, como a secagem de peças de madeira em pequenas dimensões, vem sendo testado (GIOVANELLA; MUNIZ, 2010).

Segundo Illic (1999), o pré-congelamento da madeira, pode reduzir colapsos durante a secagem da madeira e também sua retratibilidade. Entretanto, um dos principais problemas apresentados pelo método de congelamento de madeira é a possibilidade de surgir manchas na superfície desse material, desenvolver fissuras e possíveis danos causados nas paredes das células, o que pode prejudicar a sua resistência mecânica (FREITAS, 2016). Adicionalmente, o congelamento não permite a movimentação de água dentro do lume das células, dificultando a oxidação de extrativos (LIU et al., 2015), causando uma expansão do lume das células, o que pode gerar um stress nas paredes celulares, tornando-as mais rígidas, podendo reduzir as contrações da madeira.

Existem na literatura diversos trabalhos que avaliaram o uso deste prétratamento térmico. Kang et al. (2014) avaliaram o efeito do congelamento em amostras de madeira de Pseudotsuga menziesii para avaliar a taxa de secagem e os defeitos de secagem. Os autores relataram que quase não houve diferenças entre as amostras controle e as pré-congeladas em relação ao comportamento de secagem. Já Cooper (1972) relatou que o congelamento reduziu a contração de Prunus serotina Ehrh., Ulmus americana L. e Quercus alba L. 
Freitas (2016) realizou o pré-tratamento de congelamento na madeira de Eucalipto. Nos tratamentos realizados à $-20^{\circ} \mathrm{C}$, ocorreu apenas congelamento interno da água-livre presente no lume das células, gerando provavelmente colapsos internos, os quais não interferiram na resistência mecânica da madeira. Os tratamentos térmicos avaliados modificaram a estrutura dos constituintes e removeram parte dos extrativos, influenciando algumas propriedades da madeira. Segundo Taiz e Zeiger (2006), a madeira pré-congelada pode apresentar uma propensão a colapsos internos em decorrência da expansão da água, devido ao seu congelamento.

Talgatti et al. (2016) avaliaram a influência do congelamento na taxa de secagem e na qualidade da madeira de uva-do-Japão (Hovenia dulcis Thunb.). $\mathrm{O}$ congelamento da madeira possibilitou o aceleramento do processo de secagem, sendo que o tratamento de congelamento a 24 horas com as amostras orientadas no sentido tangencial apresentou maior taxa de secagem em relação as demais. As amostras não apresentaram rachaduras de topo ou de superfície em nenhum dos tratamentos avaliados no estudo. Vivian et al. (2010) também relataram que o congelamento proporcionou maiores valores de taxa de secagem de uva-do-Japão, nos tratamentos de 12 e 24 horas.

Diversos autores relataram os benefícios obtidos por meio do congelamento da madeira. Ilic (1995) e Awoyemi (2006) obtiveram uma redução da contração e dos defeitos na secagem da madeira. Já Glossop (1994) e llic (1995) relataram aumento da difusão ou permeabilidade, enquanto que Chen; Cooper (1974) e llic (1995) observaram reduções no colapso. A redução de rachaduras também foi observada (CHEN; COOPER, 1974; ILIC, 1999).

\section{CONCLUSÃO}

De uma forma geral, o uso de pré-tratamentos é benéfico para a melhoria da qualidade da madeira seca, cada um com suas especificidades. O pré-tratamento com vapor reduz a incidência de defeitos, mas tem um alto custo energético, além da possibilidade de ocorrer mudança na coloração da madeira, o que pode ser um aspecto positivo. O pré-tratamento com água quente aumenta a taxa de secagem inicial, sem ocasionar defeitos a madeira. E o pré-congelamento da madeira também 
acelera o processo de secagem, podendo haver manchas na superfície, além de fissuras e prejuízos a sua resistência mecânica.

\section{REFERÊNCIAS}

AGUIAR, O. J. R.; PERRÉ, P. Wood accelerating drying process based on its rheological properties. US n.6910284B2, 2005.

AWOYEMI, L. Influence of prefreezing on the drying and shrinkage properties of Birch (Betula pubescens) wood. Journal of the Indian Academy of Wood Science, v. 2, n. 2, p. 41-44, 2006.

CALONEGO, F. W.; SEVERO, E. T. D. Efeito da vaporização de toras na redução dos defeitos associados a tensões de crescimento de Eucalyptus grandis. Ciência Florestal, Santa Maria, v. 15, n. 4, p. 431-440, 2005.

CHAFE, S. C. Changes in shrinkage, collapse and green volume in the wood of Eucalyptus regnans F. Muell after heating in water. Wood Science, v. 12, n. 6, p. 341-345, 1992.

CHEN, P. Y. S.; COOPER, G. A. The effect of prefreezing on permeability of Black Walnut to water. Wood Science, v. 6, n. 4, p. 389-393, 1974.

CHOONG, E. T.; SHUPE, T. F.; CHEN, Y. Effect of steaming and hot-water soaking on extractive distribution and moisture diffusivity in southern pine during drying. Wood and Fiber Science, v. 31, n. 2, p. 143-150, 1999.

COOPER, G. A.; ERICKSON, R. W.; HAYGREEN, J. G. Drying behavior of prefrozen black walnut. Forest Products Journal, v. 20, n. 1, p. 30-35, 1970.

COOPER, G. A. Prefreezing reduces shrinkage and alters sorption in black walnut. Forest Products Journal, v. 22, n.5, p. 54-60, 1972.

DIAZ-VAZ, J. E.; POBLETE, H.; SCHIMIT, U. Water immersion pretreatment of Eucalyptus wood. Holzforschung, Berlin, v. 50, n. 5, p. 85-88, 1998.

ESTEVES, B. M.; PEREIRA, H. M. Wood modification by heat treatment: a review. BioResources. v.4, n.1, p. 370-404. 2009. 
FREITAS, F. P. Efeito do tratamento hidrotérmico e congelamento nas propriedades da madeira de eucalipto. 2016. Dissertação (Mestrado em Ciência Florestal). Universidade Federal de Viçosa. 51p.

GIOVANELLA, R.; MUNIZ, G. I. B. de . FLORESTA, , v. 40, n. 2, p.

311-318, 2010.

GLOSSOP, B. R. Effect of hot-water soaking or freezing pretreatments on drying rates of two eucalypts. Forest Products Journal, Madison, v. 44, n. 10, p. 29-32, 1994.

HASLETT, A. N.; KININMONTH, J. A. Pretreatments to hasten the drying of Nothofagus fusca. New Zeland Journal of Forestry Science, v.16, n.2, p.237-246, 1986

HE, X.; XIONG, X.; XIE, J. et al. Effect of Microwave Pretreatment on Permeability and Drying Properties of Wood. Bioresources, v. 12, n. 2, p, 3850-3863, 2017.

ILIC, J. Advantages of prefreezing for reducing shrinkage-related degrade in eucalypts: General considerações and review of the literature. Wood Science and Technology, v. 29, n. 4, p. 277-284, 1995.

ILIC, J. Influence of prefreezing on shrinkage related degrade in Eucalyptus regnans F. Muell. Holz als Roh und Werkstoff, v. 57, p. 241-245, 1999.

KANG, H.; HONG, S.; KANG, C. et al. Measuring Strain Variation of Pretreated Wood during Drying Using an Optical Measurement System. J. Fac. Agr., Kyushu Univ., v. 59, n. 1, p. 137-142, 2014.

KIM, Y. H.; LEE, S. M.; LEE, H. W.; LEE, J. W. Physical and chemical characteristics of products from the torrefaction of yellow poplar (Liriodendron tulipifera). Bioresour Technol. v. 116, p. 120-125, 2012.

LIU, et al. "Freezing then drying wood". BioResources, v. 10, n. 4, p. 64176427,2015

MARTINS, V. A. Secagem de madeira serrada. Brasília: IBDF, 1988. 56 p. 
MIAO, P; ZHANG, W. J. Effect of steaming treatment on transverse permeability of wood. J Nanjing Forest Univ (Nat Sci Edn), v. 33, n. 2, p. 99-102 (in Chinese with English abstract) 2009.

NUOPPONEN, M.; VUORINEN, T.; JAMSA, S. et al. The effects of heat treatment on the behaviour of extractives in softwood studied by FTIR spectroscopic methods. Wood Sci Technol. v. 37, p. 109-115, 2003.

OLIVEIRA, L. S.; SANTINI, E. J..; HASELEIN, C. R. Efeitos de pré-tratamentos de água quente e congelamento na taxa de secagem da madeira de Eucalyptus grandis Hill ex Maiden. Floresta e Ambiente, v. 10, n.2, p.11-18, 2003.

PARK, Y.; EOM, C. D.; PARK, J. H. et al. Evaluation of Physical Properties of Korean Pine (Pinus koraiensis Siebold \& Zucc.) Lumber Heat-Treated by Superheated Steam". Mokchae Konghak, v.40, n. 4, p. 257-267, 2012.

PARK, Y.; EOM, C. D.; HAN, Y.; et al. Combined treatment of green pitch pine wood by heat and superheated steam and the effects on physical properties of the products. Holzforschung, v. 68, n. 3, p. 327-335, 2014.

PARK, Y.; JANG, S.; PARK, J. et al. Changes of major chemical components in larch wood through combined treatment of drying and heat treatment using superheated steam. J Wood Sci., v. 63, p. 635-643. 2017.

PENG, Y.; LI, F.; YANG, F. et al. Effect of steam pretreatment on wood moisture content and characteristics of vacuum drying. For. Stud. China, v. 14, n. 4, p. 315319. 2012

REPELLIN, V.; GUYONNET, R. Evaluation of heat-treated wood swelling by differential scanning calorimetry in relation to chemical composition. Holzforschung, v. 59, n. 1, p. 28-34, 2005.

REZENDE, R. N.; LIMA, J. T.; RAMOS, L. E. et al. Efeito da Vaporização na secagem de tábuas de Eucalyptus grandis. Cerne. v. 21, n. 1, p. 37-43, 2015.

SEVERO, E.T.D.; TOMASELLI, I. Efeito da pré-vaporização em algumas propriedades físicas da madeira de Eucalyptus dunnii. Cerne, v. 7, n.2, p.035-042, 2001 
TALGATTI, M.; BALDIN, T.; SANTINI, E. J. et al. Tratamentos térmicos e secagem da madeira serrada de uva-do-Japão. Agrária - Revista Brasileira de Ciências Agrárias, v. 11, n.3, p. 224-229, 2016.

TAIZ, L.; ZEIGER, E. Fisiologia vegetal. São Paulo, Artmed, 3. ed., traduzida, 719 p. 2006.

TJEERDSMA, B. F.; MILITZ, H. Chemical changes in hydrothermal wood: FTIR analysis of combined hydrothermal and dry heat-treated wood. Holz Roh Werkst, v. 63, n. 2, p. 102-111, 2005.

TUONG, V. M.; LI, J. Changes caused by heat treatment in chemical composition and some physical properties of acacia hybrid sapwood. Holzforschung, v. 65, n. 1, p. 67-72, 2011.

VIVIAN, A.; BELTRAME, R.; MODES, K, S.; et al. Efeito de pré-tratamentos na taxa de secagem da madeira de Hovenia dulcis Thunb. Ciência da Madeira, v. 1, n. 2, p. 32-43. 2010.

VIVIAN, M. A.; MODES, K. S.; BELTRAME, R. Influência do tratamento térmico nos defeitos de secagem da madeira de Hovenia dulcis Thunb. Ciência da Madeira, v. 02, n. 01, p. 15-28, 2011.

WANG, Z.; YANG, X.; SUN, B.; CHAI, Y.; LIU, J.; CAO, J. Effect of vacuum heat treatment on the chemical composition of larch wood. BioResources. v. 11, n. 3, p. 5743-5750, 2016.

WIKBERG, H.; MAUNU, S. L. Characterisation of thermally modified hard- and softwoods by 13C CPMAS NMR. Carbohydr Polym. v. 58, n. 4, p. 461-466, 2004. 